\title{
Research on frequency characteristic test method of servo mechanism based on schroeder phased harmonic signal
}

\author{
Changpeng $\mathrm{Su}^{1 *}$, Xuemei Wang ${ }^{1}$, Zhe $\mathrm{Xu}^{1}$, Jianyong Wang ${ }^{2}$ and Junxiao $\mathrm{Li}^{2}$ \\ ${ }^{1}$ the Department of Automatic Control, Rocket Force University of Engineering, Xi' an, Shaanxi, 710025, China \\ ${ }^{2}$ Rocket Force Sergeant School, Qingzhou, Shandong, 262500, China
}

\begin{abstract}
The traditional frequency response test mainly adopts the sweep frequency sinusoid signal testing method, which has high accuracy, but takes a long time. This paper introduces the basic theory of the Schroeder Phased Harmonic Signal (SPHS) testing method. The comparison experiment is conducted on the servo mechanism of a certain missile, and the accuracy and rapidity of the SPHS testing method are verified. Finally, according to the experiment data, the threshold standard of SPHS parameters is put forward, which provides a reference for selecting the SPHS parameters in the frequency response test of servo mechanism.
\end{abstract}

\section{Introduction}

As the actuator of the missile attitude control system, the dynamic performance of servo mechanism directly affects the missile guidance precision. The frequency response is an important index to measure its dynamic performance, which is the main dynamic test content of servo mechanism. The traditional frequency response test mainly adopts the sweep frequency sinusoid signal method. It has high accuracy, but takes a long time, which cannot meet the requirements of accuracy and rapidity.

In recent years, some progress has been made in the fast test of frequency response. For example, in [1], Luo et al. propose that the transfer function of the system is obtained through the system identification algorithm, and the frequency response is further obtained, but the precondition is to know the order of the system model. Hao et al. introduce a frequency response test method based on the Fast Fourier Transform (FFT) algorithm in [2], but it needs to realize integral period truncation for signal, otherwise it will cause the spectrum leakage which lower test accuracy; Chen et al. use correlation analysis method for frequency response test in [3], it is simple in operation but requires computing length is an integer multiple of the signal cycle. In [4], Zhou and Li present a frequency response test method based on least square method, which uses Schroeder Phased Harmonic Signal (SPHS) as the excitation signal. Although this method can improve the test accuracy, it is easy to encounter the digital morbid problem when solving. In [5], Chen et al. give a SPHS testing method using mirror mapping method for data processing, which effectively avoids the digital morbid problem.

At present, researches on SPHS testing method only stay in mathematical simulation. In this paper, the servo mechanism of a certain missile is chosen as an object to carry out experiments, which verifies the rapidity and accuracy of the SPHS testing method. According to the safe working current of servo mechanism, the threshold standard of SPHS parameters is proposed, which provides a basis for parameter selection in the frequency response test.

\section{The schroeder phased harmonic signal}

The SPHS is a multi-frequency signal composed by a certain number of cosine waves with regular cycle, amplitude and initial phase. By adjusting the initial phase angle of cosine signals, it forms its own characteristic (see [6]). Its mathematical expression is as follows.

$$
x(t)=\sum_{k=1}^{N} \sqrt{2 P_{k}} \cos \left(\frac{2 \pi k}{T} t+\theta_{k}\right), k=1,2 \cdots N .
$$

Where $N$ is the harmonic number included in the signal, $P_{k}$ is the power of the $k$ th harmonic, $P$ is the total power of the signal, $P=\sum_{k=1}^{N} P_{k}, T$ is the cycle of the fundamental signal, $\theta_{k}$ is the initial phase of the $k$ th harmonic.

The SPHS phase is coded by Schroeder rule, which is based on the Woodward theorem( see [7]). The final formula is

$$
\theta_{k}=\pi\left[\sum_{i=1}^{k-1}(k-i) p_{i}\right], p_{i}=\frac{P_{i}}{P}
$$

Where operation $[\cdot]$ is rounded to zero, when the harmonic power is uniform, equation (2) can be simplified to: $\theta_{k}=\pi\left[\frac{k^{2}}{2 N}\right]$. In this case, the SPHS is a periodic signal, and its period is $T$. When using the Fast Fourier Transform, the whole cycle can be truncated to avoid the spectral leakage (see [8]). Moreover, the 
spectrum of the signal is discrete. It can suppress the noise interference, and realize the high-precision test.

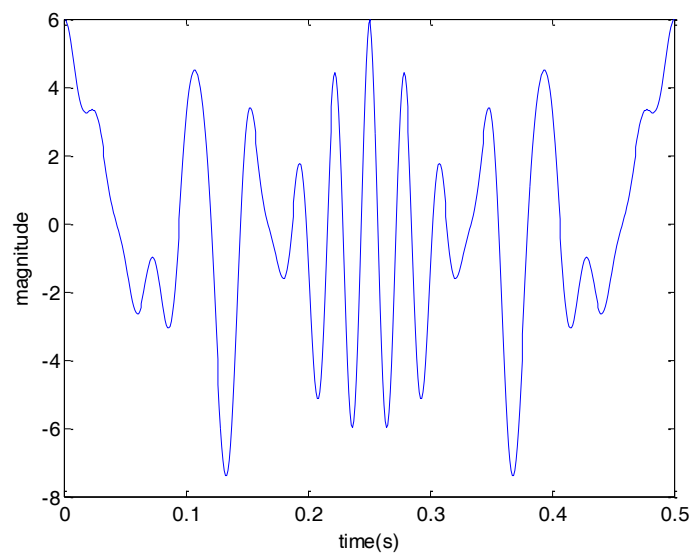

For example, the parameters of SPHS are $N=20$, $T=0.5 \mathrm{~s}, \quad P_{k}=0.5 \mathrm{~W} \quad(k=1,2, \cdots 20)$, its waveform and spectrum is shown in figure 1.

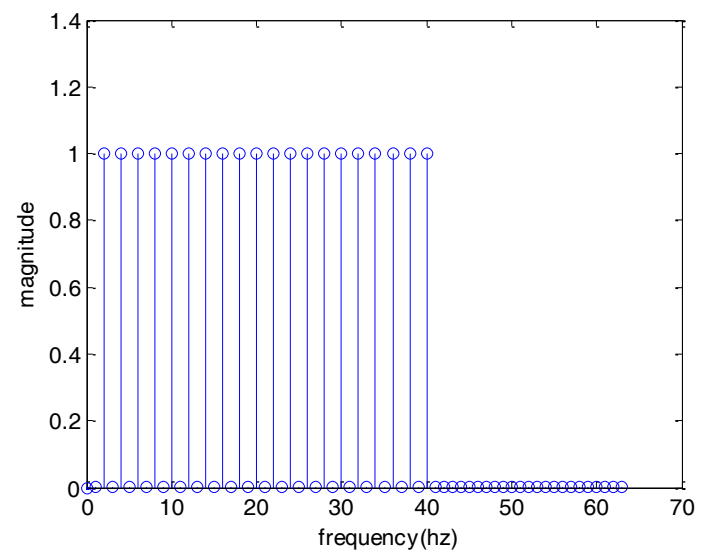

Figure 1. The waveform and spectrum of SPHS.

\section{The schroeder phased harmonic signal testing method}

When the measured system is a linear system, if the excitation is the SPHS, $x(t)=\sum_{k=1}^{N} a_{k} \cos \left(\omega_{k} t+\theta_{k}\right)$, where $\omega_{k}=2 \pi k / T, a_{k}=\sqrt{2 p_{k}}$. According to the principle of frequency invariance and superposition, the system response is as follows

$$
y(t)=\sum_{k=1}^{N} b_{k} \cos \left(\omega_{k} t+\theta_{k}+\varphi_{k}\right), k=1,2 \cdots N
$$

Where $a_{k}$ and $\theta_{k}$ are respectively the $k$ th harmonic amplitude and initial phase of the excitation signal, $b_{k}$ and $\varphi_{k}$ are respectively the $k$ th harmonic amplitude and phase shift of the system response. According to the definition of frequency characteristic, the corresponding harmonic amplitude ratio of response and excitation signals is called amplitude-frequency characteristic, $L\left(\omega_{k}\right)=20 \lg \left(b_{k} / a_{k}\right)$; the phase difference $\varphi_{k}$ is called phase-frequency characteristic.

Expanding equation (3) in accordance with the Cosine Formula, one can obtains that

$$
\begin{aligned}
y(t)= & b_{1} \cos \left(\omega_{1} t+\theta_{1}+\varphi_{1}\right)+\cdots b_{N} \cos \left(\omega_{N} t+\theta_{N}+\varphi_{N}\right) \\
= & b_{1} \cos \left(\omega_{1} t+\theta_{1}\right) \cos \left(\varphi_{1}\right)-b_{1} \sin \left(\omega_{1} t+\theta_{1}\right) \sin \left(\varphi_{1}\right)+\cdots \\
& b_{N} \cos \left(\omega_{N} t+\theta_{N}\right) \cos \left(\varphi_{N}\right)-b_{N} \sin \left(\omega_{N} t+\theta_{N}\right) \sin \left(\varphi_{N}\right) \\
& =\left[\begin{array}{c}
\cos \left(\omega_{1} t+\theta_{1}\right) \\
-\sin \left(\omega_{1} t+\theta_{1}\right) \\
\vdots \\
\cos \left(\omega_{N} t+\theta_{N}\right) \\
-\sin \left(\omega_{N} t+\theta_{N}\right)
\end{array}\right]^{T}\left[\begin{array}{c}
b_{1} \cos \left(\varphi_{1}\right) \\
b_{1} \sin \left(\varphi_{1}\right) \\
\vdots \\
b_{N} \cos \left(\varphi_{N}\right) \\
b_{N} \sin \left(\varphi_{N}\right)
\end{array}\right] .
\end{aligned}
$$

By sampling the system response, define $t_{s}$ as sample time, $n$ as sample count, equation (4) can be rewritten as follows

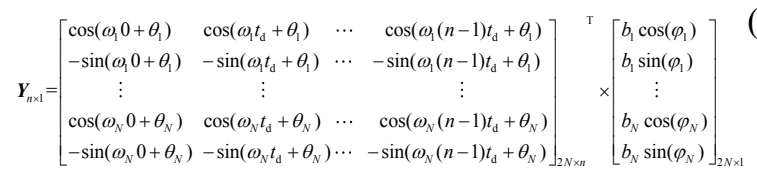

Where

$\boldsymbol{Y}^{T}=\left[y(0), y\left(t_{s}\right), y\left(2 t_{s}\right), \cdots, y\left((n-1) t_{s}\right)\right]_{1 \times n}$.

The right of equation (5) is respectively recorded as the matrix $\boldsymbol{A}_{2 N \times n}$ and vector $\boldsymbol{C}_{2 N \times 1}$, one can get that

$$
\boldsymbol{Y}_{n \times 1}=\boldsymbol{A}_{n \times 2 N}^{T} \boldsymbol{C}_{2 N \times 1} .
$$

When the sample count $n$ is large enough to make the equations number more than that of the unknown number equation (6) becomes overdetermined equations. By solving the vector $\boldsymbol{C}$, the amplitude $b_{k}$ and phase shift $\varphi_{k}$ of each harmonic in the system response can be obtained, then the frequency characteristic is further obtained.

Generally, the least square method is used to solve overdetermined equations. However, this method is easy to encounter the digital morbid problem. The mirror mapping method can avoid the digital morbid problem, and is easy to solve. The principle of mirror mapping is to use Householder Transformation to find an appropriate orthogonal matrix $\boldsymbol{H}_{n \times n}$, which makes the matrix $\boldsymbol{H}_{n \times n} \boldsymbol{A}_{n \times 2 N}^{T}$ transform into an upper triangular matrix. Define the observation equations as $\boldsymbol{L}=\boldsymbol{Y}+\boldsymbol{\Delta}$, the residual equations as $\boldsymbol{V}=\boldsymbol{A}^{T} \boldsymbol{C}-\boldsymbol{L}$, the residual sum of squares as $J=\|\boldsymbol{V}\|_{2}=\left\|\boldsymbol{A}^{T} \boldsymbol{C}-\boldsymbol{L}\right\|_{2}$. Hence the solution that minimizing the residual sum of squares is the least square solution of equation (6).

Suppose there is an orthogonal matrix $\boldsymbol{H}_{n \times n}$, one can obtains that 


$$
\boldsymbol{H}_{n \times n} \boldsymbol{A}_{n \times 2 N}^{T}=\left[\begin{array}{l}
\boldsymbol{R}_{2 N \times 2 N} \\
\boldsymbol{0}_{(n-2 N) \times 2 N}
\end{array}\right] .
$$

Define the first $2 N$ elements in the matrix $\boldsymbol{H}_{n \times n} \boldsymbol{L}$ as $\boldsymbol{m}$, and the rest as $\boldsymbol{g}$, the residual equations can be rewritten as follows

$$
\boldsymbol{H} \boldsymbol{V}=\boldsymbol{H} \boldsymbol{A}^{T} \boldsymbol{C}-\boldsymbol{H L}=\left[\begin{array}{l}
\boldsymbol{R} \\
\boldsymbol{0}
\end{array}\right] \boldsymbol{C}-\left[\begin{array}{l}
\boldsymbol{m} \\
\boldsymbol{g}
\end{array}\right]=\left[\begin{array}{c}
\boldsymbol{R} \boldsymbol{C}-\boldsymbol{m} \\
-\boldsymbol{g}
\end{array}\right] .
$$

The vector length is constant by the orthogonal transformation, so the 2-norm of equation (8) is obtained as follows

$$
J=\|\boldsymbol{V}\|_{2}=\|\boldsymbol{H} \boldsymbol{V}\|_{2}=\left\|\left[\begin{array}{c}
\boldsymbol{R} \boldsymbol{C}-\boldsymbol{m} \\
-\boldsymbol{g}
\end{array}\right]\right\|_{2}=\sqrt{(\boldsymbol{R C}-\boldsymbol{m})^{T}(\boldsymbol{R C}-\boldsymbol{m})+\boldsymbol{g}^{T} \boldsymbol{g} .}(9)
$$

When $\boldsymbol{C}=\boldsymbol{R}^{-1} \boldsymbol{m}$, the minimum residual sum of squares $J$ is $\sqrt{\mathbf{g}^{T} \boldsymbol{g}}$. Hence the least square solution of equation (6) is $\boldsymbol{C}=\boldsymbol{R}^{-1} \boldsymbol{m}$.From the vector $\boldsymbol{C}$, the amplitude ratio $L\left(\omega_{k}\right)$ and phase difference $\phi\left(\omega_{k}\right)$ corresponding to the angular frequency $\omega_{k}$ can be obtained as follows

$$
\begin{gathered}
L\left(\omega_{k}\right)=20 \lg \frac{b_{k}}{a_{k}}=20 \lg \frac{\sqrt{c^{2}{ }_{2 k-1}+c^{2}}{ }_{2 k}}{a_{k}} . \\
\phi\left(\omega_{k}\right)=\arctan \frac{c_{2 k}}{c_{2 k-1}} .(11)
\end{gathered}
$$

Where $k=1,2 \cdots N$, the above process can be expressed in figure 2 .

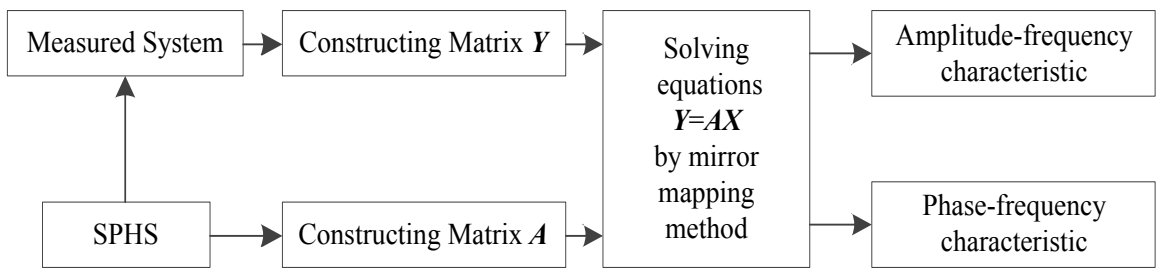

Figure 2. The flow diagram of SPHS testing method.

\section{The comparison experiment on servo mechanism}

In order to verify the testing effect of the SPHS testing method, a certain missile's servo mechanism is taken as the object, the traditional sweep frequency sinusoid signal testing method and the SPHS testing method are used respectively. The basic performance of servo mechanism is shown in table 1 .

Table 1. Working parameters of servo mechanism.

\begin{tabular}{cc}
\hline Name & Value \\
\hline Steering angle range & $\pm 20^{\circ}$ \\
Maximal working frequency & $20 \mathrm{~Hz}$ \\
Safe working current & $100 \mathrm{~A}$ \\
Settling time & $\leq 1 \mathrm{~s}$ \\
\hline
\end{tabular}

\subsection{Sweep frequency sinusoid signal testing method}

In order to perform full frequency test, the steering angle of servo mechanism should not be too large when testing. In the experiment, 3 degree is chosen as the maximal steering angle, which can test the $0-20 \mathrm{~Hz}$ frequency range. When using sweep frequency sinusoid signal testing method, 3 cycles are measured at each frequency point. It takes 300 seconds to finish the test, and the testing results are shown in table 2 .

Table 2. Testing results of frequency characteristic.

\begin{tabular}{ccc}
\hline Frequency $(\mathrm{Hz})$ & Amplitude Value $(\mathrm{dB})$ & Phase Value $\left({ }^{\circ}\right)$ \\
\hline 1.0 & -0.03 & -3.28 \\
2.5 & 0.01 & -8.28 \\
5.0 & -0.09 & -16.48 \\
7.5 & -0.34 & -24.94 \\
10.0 & -0.66 & -32.92 \\
15.0 & -1.07 & -40.48 \\
\hline
\end{tabular}




\begin{tabular}{lll}
\hline 17.5 & -1.57 & -47.70 \\
20.0 & -2.10 & -54.54 \\
\hline
\end{tabular}

Based on the above data, the frequency characteristic is obtained and shown in figure 3 .
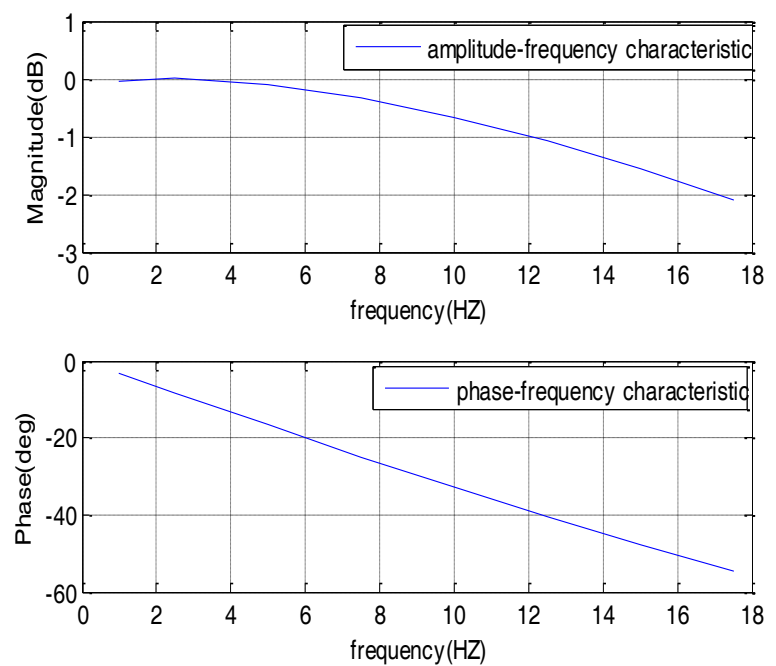

Figure 3. Frequency response obtained by sweep sinusoid testing method.

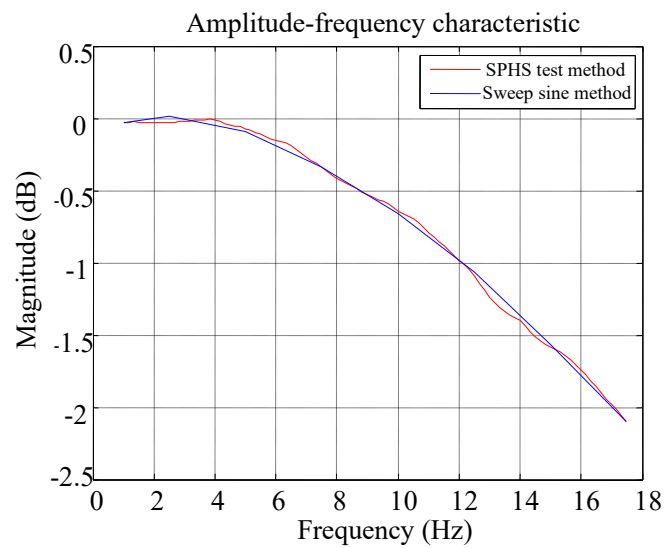

\subsection{The SPHS testing method}

When using the SPHS testing method, in order to ensure the stability of the signal and the reliability of the testing data, two periods of SPHS are inputted into the servo mechanism, and the second cycles of the feedback signal is selected as the experimental result. It takes 100 seconds to finish the testing, with the data processed by the mirror mapping method. The frequency response of servo mechanism is shown in figure 4 .

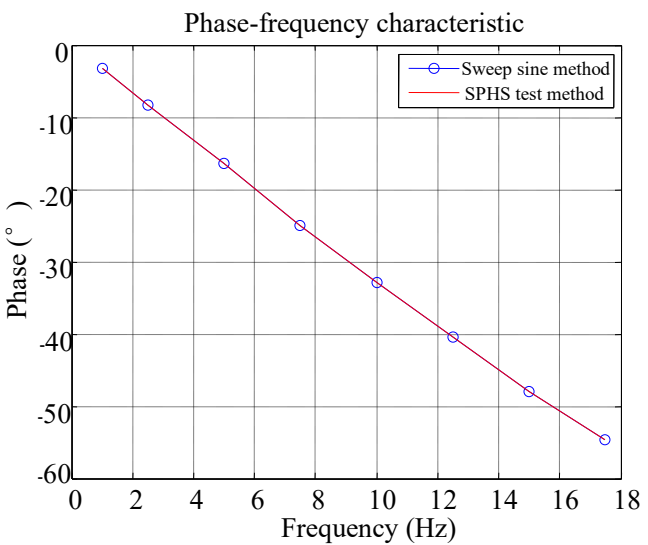

Figure 4. Comparison of frequency response measured by two methods.

\subsection{Testing performance comparison}

The results obtained by two testing methods are compared with the designed value at the critical frequency points, which is shown in table 3 . The average error of amplitude-frequency characteristic measured by the sweep frequency sinusoid signal testing method is about $0.04 \mathrm{~dB}$, that measured by the SPHS testing method is about $0.032 \mathrm{~dB}$. Therefore, the SPHS testing method is more accurate.

Table 3. The testing results comparison.

\begin{tabular}{cccccc}
\hline Frequency $(\mathrm{Hz})$ & 1.0 & 5.0 & 10.0 & 15.0 & 20.0 \\
\hline Designed Value $(\mathrm{dB})$ & -0.025 & -0.088 & -0.652 & -1.243 & -2.115 \\
SPHS Test Method $(\mathrm{dB})$ & -0.03235 & -0.08736 & -0.6583 & -1.1102 & -2.1018 \\
Sweep Sinusoid Method(dB) & -0.03 & -0.09 & -0.66 & -1.07 & -2.1 \\
\hline
\end{tabular}


In the aspect of testing time, it takes 300 seconds by the sweep frequency sinusoid signal testing method, and 100 seconds by the SPHS testing method. The testing time is reduced by $66.7 \%$ compared with the sweep frequency sinusoid signal testing method.

When the tested angle increases, the tested frequency band must be reduced. Based on the experiments on the threshold of tested frequency and angle, considering the safe working current of servo mechanism, it is summarized the threshold standard of SPHS parameters when the fundamental period $T$ is 5 seconds, as is shown in table 4

Table 4. Threshold standard of SPHS parameters.

\begin{tabular}{cccccc}
\hline Power Threshold $(\mathrm{W})$ & 6.65 & 12.25 & 17.5 & 29.5 & 75 \\
\hline Harmonic Number & 100 & 90 & 60 & 50 & 10 \\
Frequency Band $(\mathrm{Hz})$ & 20 & 18 & 12 & 5 & 2 \\
Tested Angle $\left(^{\circ}\right)$ & 1 & 3 & 5 & 10 & 20 \\
\hline
\end{tabular}

\section{Conclusion}

The experimental results show that the SPHS test method can achieve the rapid and high-precision test of the frequency characteristic, and it is easy to conduct. In the light of the problem that it is difficult to execute full frequency test in large-angle state, the threshold standard of parameters $T, N, P$ for SPHS has been made through the experiment, which provides a reference for selecting the parameters of SPHS in the frequency characteristic test of servo mechanism.

\section{References}

1. Luo, D.C., Wang, S.C., Liu, Z.G. (2015) Application of household in the frequency domain modeling of the lateral-normal stabilizer of missile. Journal of Projectiles; Rockets; Missiles and Guidance, 25: 614-616.

2. Hao, W.L., Li, F.H., Xu, H.L. (2005) Frequency Domain Modeling Method Excited by SPHS and Matlab. China Measurement Technology, 31: $118-120$.

3. Chen, L.H., Zhao, N., Wang, Y.B. (2011) Fast Frequency Response Testing Method with One Step. Electronic Measurement Technology, 34: 77-79.

4. Zhou, J.B., Li, J. (2016) Frequency Response Testing Method Based on SPHS Signal. Industrial Control Computer, 29: 39-41.

5. Chen, L.H., Yue, R.H., Zhao, N. (2011) Frequency Response Testing Method Based on the Schroeder-phased Harmonic Signal. Theory and Method, 30: 13-15.

6. Zhu, Y.M., Hou, W., Zhang, Y. (2018) Schroeder Phased Harmonic Signal Applied in Frequency Response Test of Servo System. Chinese journal of Electron Devices, 39: 35-41.

7. Schroeder, M.R. (1970) Synthesis of Low-PeakFactor Signals and Binary Signals with Low Autocorrelation. IEEE Trans Information Theory, 85-89.

8. Van den Bos, A., Krol, R.G. (1979) Synthesis of
Discrete-Interval Binary Signals with Specified Fourier Amplitude Spectra. International Journal of Control, 30: 871-884. 